\title{
Recomendaciones perioperatorias y transoperatorias para la prevención de infecciones del sitio quirúrgico en ortopedia
}

\author{
Perioperative and transoperative recommendations for the prevention \\ of surgical site infections in orthopedics
}

Antonio Ramos de la Medina, ${ }^{*}$ María José Martínez Lara ${ }^{\ddagger}$

\footnotetext{
${ }^{*}$ Cirujano General, Hospital de la Beneficencia Española, Veracruz Ver. Director del Centro de Investigación en Cirugía Global [Global Health Research Unit on Global Surgery (NIHR)]. Hospital Español Veracruz.

‡ Médico Pasante en Servicio Social Universidad Autónoma de Veracruz-Villa Rica. Hospital Naval de Tampico.
}

Correspondencia: Dr. Antonio Ramos de la Medina. Hospital Español Veracruz, 16 de Septiembre No. 955, Centro, 91700, Veracruz, Ver. Correo electrónico: ramos.antonio@heveracruz.mx

\section{RESUMEN}

Existen diferentes medidas para mejorar las condiciones de los pacientes programados electivamente o por urgencia para cirugía ortopédica o traumatológica. En esta revisión abordaremos las medidas perioperatorias y transoperatorias y su influencia en la prevención de la infección del sitio quirúrgico en Ortopedia. Trataremos de resumir evidencia de calidad de manera breve.

Palabras clave: Prevención, infección del sitio quirúrgico, cirugía ortopédica, condiciones perioperatorias, periodo transoperatorio.

\section{ABSTRACT}

There are different measures to improve the conditions of patients scheduled electively or by emergency for orthopedic or trauma surgery. In this review we will address perioperative and transoperative measures and their influence on the prevention of surgical site infection in Orthopaedics. We will try to summarize quality evidence briefly.

Keywords: Prevention, surgical site infection, orthopedic surgery, perioperative conditions, transoperative period.

\section{APOYO NUTRICIONAL}

La desnutrición, incluidas las deficiencias calórico-proteicas y de micronutrientes, sigue siendo un importante problema de salud pública, especialmente en los países en vías de desarrollo; además, afecta a la población de la tercera edad en rápido crecimiento en los países de altos ingresos. ${ }^{1}$ El estado nutricional podría tener un profundo impacto en el sistema inmune según lo documentado por algunos estudios. Estas alteraciones en la inmunidad del huésped pueden hacer que los pacientes sean más susceptibles a las infecciones postoperatorias y la desnutrición se reportó como una amenaza para el resultado quirúrgico, tal 
como recuperación tardía, mayores tasas de morbilidad y mortalidad, estadía prolongada en el hospital, aumento de los costos de atención médica y una mayor tasa de reingreso temprano. ${ }^{2}$

Algunos estudios mostraron que el soporte nutricional temprano puede mejorar el resultado después de una cirugía mayor y disminuir la incidencia de complicaciones infecciosas en pacientes seleccionados desnutridos o con lesiones graves. La evidencia muestra que una fórmula nutricional mejorada con múltiples nutrientes tiene un beneficio significativo en comparación con una fórmula nutricional estándar para reducir el riesgo de infección del sitio quirúrgico. ${ }^{3}$

\section{INTERRUPCIÓN PERIOPERATORIA DE FÁRMACOS INMUNOSUPRESORES}

Hasta la fecha, únicamente ha sido publicada una guía por la Sociedad de Epidemiología Sanitaria de América y la Asociación de Enfermedades Infecciosas de América, y ésta recomienda evitar el uso de agentes inmunosupresores en el periodo perioperatorio si es posible. ${ }^{4}$

Al tener en cuenta la evidencia muy limitada para el antifactor de necrosis tumoral o la falta de evidencia e incluso el daño potencial del metrotexato que apoyar la interrupción del tratamiento, y el riesgo asociado con la interrupción del tratamiento en el paciente con enfermedad subyacente, la medicación inmunosupresora no debe suspenderse con el fin de prevenir infección en el sitio quirúrgico. ${ }^{5}$

\section{OXIGENACIÓN PERIOPERATORIA}

Se recomienda que los pacientes adultos sometidos a anestesia general con intubación endotraqueal para procedimientos quirúrgicos reciban una fracción de $80 \%$ de oxígeno inspirado $\left(\mathrm{FiO}_{2}\right)$ intraoperatoriamente y, si es posible, en el postoperatorio inmediato durante dos a seis horas para reducir el riesgo de SSI. Existe evidencia de que un flujo sanguíneo optimizado hacia la incisión quirúrgica redujo las tasas de infección de herida quirúrgica al evitar la hipotermia, la hipoxia y la disminución de la perfusión. ${ }^{6}$ Desde el año 2000 se han publicado varios ensayos sobre el uso de altas concentraciones de $\mathrm{FiO}_{2}$ durante el periodo perioperatorio y la posible asociación con tasas más bajas de infección de sitio quirúrgico.

Un estudio comparó la $\mathrm{FiO}_{2}$ perioperatoria aumentada (80\%) con la $\mathrm{FiO}_{2}$ estándar (30-35\%) en pacientes sometidos a procedimientos bajo anestesia general con intubación endotraqueal y mostró que el aumento de $\mathrm{FiO}_{2}$ perioperatorio es beneficioso para reducir la infección del sitio quirúrgico (ISQ) en comparación con la $\mathrm{FiO}_{2}$ perioperatoria estándar en esta población de pacientes. ${ }^{7}$

La Sociedad de Epidemiología Sanitaria de América y la Asociación de Enfermedades Infecciosas de América recomiendan optimizar la oxigenación de los tejidos mediante la administración de oxígeno suplementario durante e inmediatamente después de los procedimientos quirúrgicos que involucran ventilación. ${ }^{6}$ 


\section{MANTENIMIENTO DE LA NORMOTERMIA}

Los eventos más comunes que derivan a hipotermia son la exposición a un ambiente frío en la sala de operaciones y el deterioro inducido por la anestesia del control termorregulador. La exposición de la piel durante el periodo perioperatorio puede aumentar la pérdida de calor. Además, los líquidos por vía intravenosa y de irrigación enfrían directamente a los pacientes. Los sedantes y los agentes anestésicos inhiben la respuesta normal al frío, lo que mejora el flujo sanguíneo hacia la periferia y aumenta la pérdida de calor. ${ }^{8}$

No está claro cómo el mantenimiento de la normotermia en el compartimento central del cuerpo podría reducir la incidencia de ISQ. Todos los estudios disponibles miden la temperatura central y no periférica. Sin embargo, es muy probable que las temperaturas centrales más bajas reportadas den como resultado una temperatura cutánea reducida en el sitio operatorio. No obstante, no se ha demostrado que el calentamiento incisional disminuya las tasas de infección del sitio quirúrgico. ${ }^{9}$

\section{USO DE PROTOCOLOS PARA EL CONTROL INTENSIVO DE LA GLUCEMIA PERIOPERATORIA}

Varios estudios observacionales mostraron que la hiperglucemia se asocia con un mayor riesgo de SSI y, por lo tanto, un mayor riesgo de morbilidad, mortalidad y mayores costos de atención médica en pacientes diabéticos y no diabéticos, así como en diferentes tipos de cirugía. Además, algunos estudios que apuntan a niveles de glucosa perioperatoria relativamente bajos han resaltado el riesgo de efectos adversos asociados con protocolos intensivos, ya que pueden causar hipoglucemia con un grave riesgo de complicaciones mortales..$^{10,11}$

Varias organizaciones han emitido recomendaciones sobre el control de la glucemia perioperatoria. Si bien la mayoría de las recomendaciones se centran sólo en el paciente diabético, las emitidas por la Sociedad de Epidemiología de América (SHEA)/Sociedad de Enfermedades Infecciosas de América (IDSA) ${ }^{12}$ y el Colegio Americano de Médicos (ACP $)^{13}$ se aplican a todos los pacientes quirúrgicos. Recomiendan niveles objetivo entre 140-200 mg/dl (7.8-11.1 mmol/l) o límites superiores de $180 \mathrm{mg} / \mathrm{dl}(10 \mathrm{mmol} / \mathrm{l})$ o $198 \mathrm{mg} / \mathrm{dl}(11 \mathrm{mmol} / \mathrm{l}){ }^{4}$

\section{MANTENIMIENTO DE LA NORMOVOLEMIA}

En la actualidad, no existe una definición universal de normovolemia o un método estandarizado para su evaluación. Las pautas SHEA/IDSA no formulan una recomendación específica sobre el mantenimiento de la normovolemia para la prevención de SSI. Sin embargo, en una declaración sobre oxigenoterapia, se recomienda indirectamente mantener un reemplazo de volumen apropiado. La Organización Mundial de la Salud (OMS) argumentó que tanto la sobrecarga de líquidos como la hipovolemia pueden aumentar la mortalidad y morbilidad. ${ }^{4,14}$ 


\section{CAMPOS Y BATAS}

Durante los procedimientos quirúrgicos el riesgo de transmisión de patógenos aumenta si los materiales de barrera se mojan. En consecuencia, los materiales de uso múltiple o único de los campos y batas utilizados en un procedimiento quirúrgico deben evitar la penetración de líquidos. Los materiales reutilizables se componen típicamente de diferentes tejidos (algodón, tejidos posiblemente mezclados con poliéster y/o tratados químicamente), estos productos tienen que ser duraderos y proporcionar protección después de muchos ciclos de procesamiento y tratamiento. Por lo general, los campos y batas quirúrgicas desechables no están compuestas de tejido, pueden ser de origen sintético y/o natural, posiblemente combinado con tratamientos químicos. ${ }^{15}$

Todo esto debe tenerse en cuenta al evaluar las implicaciones de los recursos, ya que éstos incluyen los costos de compra directa y los costos relacionados con la lavandería y la esterilización, la mano de obra requerida para el procesamiento y la eliminación de desechos. Dos estudios mostraron costos más bajos asociados con el uso de campos y batas desechables, mientras que un análisis de costo-beneficio encontró que los costos de los campos y batas desechables estériles son relativamente más altos en comparación con los reutilizables. Otros autores informaron que los costos eran similares para los artículos desechables y reutilizables. ${ }^{16,17}$ Los hallazgos heterogéneos de los datos disponibles sobre las implicaciones de los recursos sugieren que los campos y batas quirúrgicas desechables y reutilizables son probablemente similares en costos.

\section{APÓSITOS PROTECTORES DE HERIDAS}

Teóricamente, los protectores de heridas disponibles comercialmente están destinados a reducir la contaminación del borde de la herida al mínimo durante los procedimientos quirúrgicos, incluida la contaminación del exterior (cirugía limpia) y del interior de la cavidad peritoneal (cirugía limpia, contaminada, contaminada y sucia).

En artroplastias de rodilla y cadera, un apósito hidrocoloide impregnado con plata demostró disminuir la tasa de infecciones del sitio quirúrgico en comparación con apósitos convencionales hasta en $76 \% .{ }^{18} \mathrm{En}$ el caso de fracturas expuestas, el uso de terapia de presión negativa en comparación con apósitos convencionales resultó en una menor tasa de infección, menor tiempo de cierre de la herida y menor tiempo de hospitalización. ${ }^{19}$

\section{IRRIGACIÓN INTRAOPERATORIA DE LA HERIDA}

Las Guías de la OMS consideran que no hay pruebas suficientes para recomendar a favor o en contra de la irrigación salina de las heridas incisionales antes del cierre con el fin de prevenir la ISQ, sugieren considerar el uso de la irrigación de la herida con una solución acuosa de povidona yodada al $1 \%$ antes del cierre con el fin de prevenir infecciones del sitio quirúrgico. En un análisis reciente de 11,738 
casos de artroplastias que fueron irrigadas con iodopovidona antes del cierre de la herida, no se encontraron diferencias significativas en la tasa de infecciones del sitio quirúrgico. ${ }^{20}$ En otro estudio de artroplastias de cadera y rodilla en 253 pacientes, compararon la irrigación de la herida quirúrgica durante y antes del cierre con diferentes soluciones: solución salina, iodopovidona y clorhexidina. Ninguna solución demostró ventajas sobre las otras para disminuir la tasa de infección del sitio quirúrgico. ${ }^{21}$

\section{USO DE GUANTES QUIRÚRGICOS}

Una revisión Cochrane publicada en 2009 investigó si la protección adicional del guante reduce la cantidad de ISQ o infecciones transmitidas vía sanguínea en pacientes o en el equipo quirúrgico y el número de perforaciones en el par más interno de guantes quirúrgicos. No hubo evidencia directa de que la protección adicional del guante usada por el equipo quirúrgico reduzca la ISQ en los pacientes. Sin embargo, la revisión tuvo un poder insuficiente para este resultado, ya que sólo se encontraron dos ensayos con el resultado primario de ISQ, los cuales no informaron infecciones. No se encontraron ensayos con infecciones transmitidas por la sangre como resultado en pacientes quirúrgicos o en el equipo quirúrgico en relación con el método de guantes. ${ }^{22}$ Se identificaron 31 estudios de control aleatorizados con el resultado de la perforación del guante, lo que condujo a la conclusión de que el uso de un segundo par de guantes quirúrgicos, guantes triples, guantes externos de punto y forros de guantes reduce significativamente las perforaciones en los guantes más internos.

Pocas organizaciones han emitido recomendaciones sobre el uso de guantes, las últimas pautas de la OMS para cirugía segura, publicadas en 2009, recomiendan que el equipo quirúrgico se cubra el cabello y use batas y guantes estériles durante la operación, pero sin ninguna indicación de guantes simples o dobles. Además, existe evidencia que sustenta el cambio frecuente de guantes externos para disminuir el riesgo de infecciones. ${ }^{23}$

\section{SUTURAS RECUBIERTAS CON ANTIMICROBIANOS}

Las suturas recubiertas disponibles comercialmente con propiedades antimicrobianas son la poliglactina 910 recubierta con triclosán, la polidioxanona recubierta con triclosán y la poliglecaprona 25 recubierta con triclosán. Las suturas no recubiertas de uso común son poliglactina 910 , polidioxanona, poliglecaprona 25 , ácido poliglicólico y suturas de poligluconato.

Varios ensayos han demostrado que el uso de suturas recubiertas con triclosán conduce a una reducción del número de bacterias in vitro y también de infecciones de heridas en estudios clínicos y animales. En general, existe evidencia de que las suturas con recubrimiento antimicrobiano tienen un beneficio significativo en la reducción de las tasas de ISQ en pacientes sometidos a procedimientos quirúrgicos en comparación con las suturas sin recubrimiento. ${ }^{24-26}$ 


\section{SISTEMAS DE VENTILACIÓN DE FLUJO DE AIRE LAMINAR EN EL CONTEXTO DE LA VENTILACIÓN EN QUIRÓFANO}

El sistema de ventilación en la sala de operaciones está diseñado principalmente para crear comodidad térmica para el paciente y el personal, y para mantener una calidad de aire constante al eliminar aerosoles y partículas dentro de la sala. Se requieren sistemas especiales de ventilación que suministren aire filtrado a presión positiva en la sala quirúrgica. Idealmente, se necesitan alrededor de 20 cambios de aire por hora para diluir los microorganismos generados en la sala de operaciones y para excluir el ingreso de las áreas circundantes. ${ }^{12,13}$

En entornos con buenos recursos, los sistemas de ventilación convencional que hacen pasar aire con un flujo mixto o turbulento al quirófano son los más instalados. Esto conduce a una dilución acelerada del volumen de aire y a un movimiento irregular de las partículas. Los sistemas de ventilación turbulenta convencionales se utilizan para todo tipo de cirugía. Los sistemas con ventilación de flujo laminar se ocupan con frecuencia en un entorno donde la contaminación con partículas es un evento altamente adverso, por ejemplo, la cirugía de implante ortopédico. El objetivo de pasar el aire fresco unidireccionalmente con una velocidad constante y líneas de corriente aproximadamente paralelas es crear una zona donde se expulse el aire, los aerosoles y las partículas dentro de la habitación. Las limitaciones a este principio son todas las fuerzas que interrumpen el flujo de aire paralelo. Sin embargo, no han probado disminuir la incidencia de infecciones del sitio quirúrgico en comparación con sistemas convencionales de ventilación..$^{27-29}$ Por otro lado, el mantener cerradas las puertas del quirófano disminuye la turbulencia en el mismo y la tasa de crecimiento bacteriano en controles establecidos dentro y fuera del área de ventilación de flujo laminar. ${ }^{30}$

\section{BIBLIOGRAFÍA}

1. Muller O, Krawinkel M. Malnutrition and health in developing countries. CMAJ. 2005; 173 (3): $279-286$.

2. Mainous MR, Deitch EA. Nutrition and infection. Surg Clin North Am. 1994; 74 (3): 659-676.

3. He Y, Xiao J, Shi Z, He J, Li T. Supplementation of enteral nutritional powder decreases surgical site infection, prosthetic joint infection, and readmission after hip arthroplasty in geriatric femoral neck fracture with hypoalbuminemia. J Orthop Surg Res. 2019; 14 (1): 292.

4. Anderson DJ, Podgorny K, Berrios-Torres SI, Bratzler DW, Dellinger EP, Greene L, et al. Strategies to prevent surgical site infections in acute care hospitals: 2014 update. Infect Control Hosp Epidemiol. 2014; 35 (6): 605-627.

5. Pountos I, Giannoudis PV. Effect of methotrexate on bone and wound healing. Expert Opin Drug Saf. 2017; 16 (5): 535-545.

6. Kurz A, Fleischmann E, Sessler DI, Buggy DJ, Apfel C, Akca O. Effects of supplemental oxygen and dexamethasone on surgical site infection: a factorial randomized trial doublé dagger. Br $\mathrm{J}$ Anaesth. 2015; 115 (3): 434-443.

7. Stall A, Paryavi E, Gupta R, Zadnik M, Hui E, O'Toole RV. Perioperative supplemental oxygen to reduce surgical site infection after open fixation of high-risk fractures: a randomized controlled pilot trial. J Trauma Acute Care Surg. 2013; 75 (4): 657-663.

8. Sessler DI. Mild perioperative hypothermia. New Engl J Med. 1997; 336 (24): 1730-1737.

9. Diaz M, Becker DE. Thermoregulation: physiological and clinical considerations during sedation and general anesthesia. Anesthes Prog. 2010; 57 (1): 25-32; quiz 3-4.

10. Ata A, Lee J, Bestle SL, Desemone J, Stain SC. Postoperative hyperglycemia and surgical site infection in general surgery patients. Arch Surg. 2010; 145 (9): 858-864. 
11. Kao LS, Phatak UR. Glycemic control and prevention of surgical site infection. Surg Infect (Larchmt). 2013; 14 (5): 437-444.

12. Sehulster L, Chinn RY. Guidelines for environmental infection control in health-care facilities. Recommendations of CDC and the Healthcare Infection Control Practices Advisory Committee (HICPAC). Morbid Mortal Wkly Rep. 2003; 52 (Rr-10): 1-42.

13. Atkinson JYC, Pessoa-Silva CL, Jensen P, Li Y, Seto WH. Natural ventilation for infection control in health-care settings. Geneva: World Health Organization; 2009.

14. Venn R, Steele A, Richardson P, Poloniecki J, Grounds M, Newman P. Randomized controlled trial to investigate influence of the fluid challenge on duration of hospital stay and perioperative morbidity in patients with hip fractures. Br J Anaesth. 2002; 88 (1): 65-71.

15. Murphy L. Cost/benefit study of reusable and disposable OR draping materials. J Healthc Mat Manage. 1993; 11 (3): 44-48.

16. Lizzi AM, Almada GC, Veiga G, Carbone N. Cost effectiveness of reusable surgical drapes versus disposable non-woven drapes in a Latin American hospital. Am J Infect Control. 2008; 36 (5): e125.

17. Overcash M. A comparison of reusable and disposable perioperative textiles: sustainability state-ofthe-art 2012. Anesth Analg. 2012; 114 (5): 1055-1066

18. Grosso MJ, Berg A, LaRussa S, Murtaugh T, Trofa DP, Geller JA. Silver-impregnated occlusive dressing reduces rates of acute periprosthetic joint infection after total joint arthroplasty. J Arthroplasty. 2017; 32 (3): 929-932.

19. Liu X, Zhang H, Cen S, Huang F. Negative pressure wound therapy versus conventional wound dressings in treatment of open fractures: a systematic review and meta-analysis. Int J Surg. 2018; 53: 72-79.

20. Hernandez NM, Hart A, Taunton MJ, Osmon DR, Mabry TM, Abdel MP, Perry KI. Use of povidone-iodine irrigation prior to wound closure in primary total hip and knee arthroplasty: an analysis of 11,738 cases. J Bone Joint Surg Am. 2019; 101 (13): 1144-1150

21. Frisch NB, Kadri OM, Tenbrunsel T, Abdul-HakA, Qatu M, Davis JJ. Intraoperative chlorhexidine irrigation to prevent infection in total hip and knee arthroplasty. Arthroplast Today. 2017; 3 (4): 294-297.

22. Tanner J, Parkinson H. Double gloving to reduce surgical cross-infection. Cochrane Database Syst Rev. 2006; (3): CD003087.

23. Ward WG Sr, Cooper JM, Lippert D, Kablawi RO, Neiberg RH, Sherertz RJ. Glove and gown effects on intraoperative bacterial contamination. Ann Surg. 2014; 259 (3): 591-597.

24. Marco F, Vallez R, Gonzalez P, Ortega L, de la Lama J, Lopez-Duran L. Study of the efficacy of coated Vicryl plus antibacterial suture in an animal model of orthopedic surgery. Surg Infect (Larchmt). 2007; 8 (3): 359-365.

25. Rothenburger S, Spangler D, Bhende S, Burkley D. In vitro antimicrobial evaluation of coated VICRYL* Plus antibacterial suture (coated polyglactin 910 with triclosan) using zone of inhibition assays. Surg Infect (Larchmt). 2002; 3 (Suppl. 1): S79-87.

26. Storch ML, Rothenburger SJ, Jacinto G. Experimental efficacy study of coated VICRYL plus antibacterial suture in guinea pigs challenged with Staphylococcus aureus. Surg Infect (Larchmt). 2004; 5 (3): 281 288.

27. McHugh SM, Hill AD, Humphreys H. Laminar airflow and the prevention of surgical site infection. More harm than good? Surgeon. 2015; 13 (1): 52-58.

28. Erichsen Andersson A, Petzold M, Bergh I, Karlsson J, Eriksson BI, Nilsson K. Comparison between mixed and laminar airflow systems in operating rooms and the influence of human factors: experiences from a Swedish orthopedic center. Am J Infect Control. 2014; 42 (6): 665-669.

29. Brandt C, Hott U, Sohr D, Daschner F, Gastmeier P, Rüden H. Operating room ventilation with laminar airflow shows no protective effect on the surgical site infection rate in orthopedic and abdominal surgery. Ann Surg. 2008; 248 (5): 695-700.

30. Perez P, Holloway J, Ehrenfeld L, Cohen S, Cunningham L, Miley GB, et al. Door openings in the operating room are associated with increased environmental contamination. Am J Infect Control. 2018; 46 (8): 954-956. 